(hrGFP), as well as null-content adenoviral vector, was prepared. Swine of both genders were percutaneous intracoronary injected with adenoviral vector expressing ILK or empty ad-null in left anterior descending coronary artery (LAD) following left anterior descending coronary artery occlusion. ILK and report gene (hrGFP) expression were confirmed by western blotting and immunohistochemistry in both noninfarcted and infarcted hearts. Echocardiographic and PET-CT/SPECT analyses were performed 4 weeks after transfection. Then myocardial tissues were harvested and fixed for subsequent histological, immunohistochemical and TUNEL examination. Histological analysis of left ventricle was performed using $\mathrm{H} \& \mathrm{E}$ staining. Angiogenesis was evaluated by microvessel density using vwf staining. Apoptosis was measured by TUNEL analysis. Cardiomyocyte proliferation was estimated by Proliferating Cell Nuclear Antigen, phosphohistone-H3 staining. Moreover, we cultivated neonate rat cardiomyocyte in hypoxia condition and observed cardiomyocyte proliferation apoptosis as well as expression of VEGF after transfered ad-ILK.

Results Western blottings and immunohistochemistry analysis revealed higher expression of ILK and hrGFP in infarct area of ad-ILK heart compared with ad-null controls after 4 week adenoviral delivery, The exotic gene was mainly expressed in cardiomyocytes and partly in cardiac fibroblasts. Four weeks after transfection, echocardiographic and PET-CT/SPECT analysis demonstrated relatively preserved cardiac function in the ILK group. ILK treatment was associated with reduced infarct scar size, preserved LV geometry (including LV diameter, LV wall thickness, cardiomyocyte size). Enhanced angiogenesis was oberserved in ad-ILK animals. TUNEL analysis also revealed a reduction in apoptosis in the ILK group. Moreover, in vitro ILK increased cardiomyocyte proliferation was found through phospho-histone H3, decreased cardiomyocyte apoptosis, and increased VEGF expression.

Conclusions ILK gene therapy improves cardiac remodelling and function in swines following myocardial infarction, and is associated with increased angiogenesis, reduced apoptosis and increased cardiomyocyte proliferation. These results may deliver a new approach to the treatment of post-infarct remodelling and subsequent heart failure.

\section{e0432 AN ESSENTIAL ROLE OF SERUM B-TYPE NATRIURETIC PEPTIDE IN PATIENTS WITH ACUTE INFERIOR MYOCARDIAL INFARCTION}

doi:10.1136/hrt.2010.208967.432

${ }^{1}$ Chen Ling, ${ }^{2} Y_{\text {in Xihu, }}{ }^{2}$ Luo Yun, ${ }^{1}$ Hu Jianxin, ${ }^{1}$ Hong Kui. ${ }^{1}$ The Second Affiliated Hospital Of Nanchang University; ${ }^{2}$ The First People's Hospital Jiujiang

Objective To investigate the relationship between the level of serum B-type natriuretic peptide (BNP) and right ventricular infarction in patient $\mathrm{s}$ with acute inferior myocardial infarction (AIMI).

Method The serum BNP level was measured in 213 consecutive patient $\mathrm{s}$ with AIMI who were admitted in the coronary care unit (CCU) and in the normal control with 98 cases from October 2006 to May 2009. The patients were divided into five groups in accordance with the coronary angiographic findings and ECG: Acute inferior myocardial infarction group; acute inferior myocardial infarction and right ventricular infarction group; control group; left anterior circumflex (LXC) group; proximal, middle and distal segment of right coronary artery (RCA)group. All patient s were performed directly percutaneous coronary intervention (PCI) within $24 \mathrm{~h}$ after the onset of AIMI. The incidence of major adverse cardiac events (MACE), including arrhythmia, heart failure, cardiac shock and mortality, had been observed during hospital, 30 days and 3 months.
Result BNP level in the acute inferior myocardial infarction and right ventricular infarction group was significantly higher than that in acute inferior myocardial infarction group $(p<0.101)$. The level in the proximal2mid segment of RCA group was higher than that in the LXC group $(p<0.101)$. Additionally, logistic regression analysis showed that the level of BNP was an independent predictor of MACE in the 30 days and 3 months in acute inferior myocardial infarction patient $\mathrm{s}(\mathrm{r}=0.7010,95 \% \mathrm{CI}<0.01$ to 0.615 , $\mathrm{p}<0.101)$.

Conclusion We demonstrated the level of BNP in patients with acute inferior myocardial infarction or/and right ventricular infarction, and BNP could be a good predictor for patients with acute inferior myocardial infarction and right ventricular infarction.

\section{e0433 EFFECT OF ATROVASTATIN THERAPY ON BORDERLINE VULNERABLE LESIONS IN PATIENTS WITH ACUTE CORONARY SYNDROME}

doi:10.1136/hrt.2010.208967.433

${ }^{1} Y_{u}$ Danqing, ${ }^{2}$ Lin Shuguang, ${ }^{3}$ Chen Jiyan, ${ }^{4}$ Xue Ling, ${ }^{2}$ Li Guang, ${ }^{3}$ Dong Haojian. ${ }^{1}$ Guangdong Provincial Cardiovascular Research Institute, Guangdong Provincial Academy Of Medical Science, Guangdong Provincial People's Hospital, Guangzhou; ${ }^{2}$ Guangdong Provincial Cardiovascular Research Institute; ${ }^{3}$ Guangdong Provincial Academy Of Medical Science; ${ }^{4}$ Guangdong Provincial People's Hospital

Objective To evaluate the effect of atrovastatin therapy on borderline vulnerable lesions in patients with acute coronary syndrome (ACS) and to investigate the relationship between lesion reversion and the level of MMP-9, TIMP-1, hs-CRP respectively.

Methods Patients with ACS whose serum LDL-C is lower than 2.1 $\mathrm{mmol} / \mathrm{L}$ underwent coronary angiography (CAG) and intravascular ultrasound (IVUS) investigation. If the culprit lesions were demonstrated to be borderline lesions (coronary artery stenosis between $50-70 \%$ ) by CAG and minimal lumen cross-sectional area (CSA) $>4.0 \mathrm{~mm}^{2}$ by IVUS, the patients were enrolled in the present study. Intravascular ultrasound was performed to assess coronary atheroma at baseline and 12 months after atrovastatin therapy. The level of MMP-9, TIMP-1, hs-CRP were respectively measured by ELISA at baseline and 12 month-follow-up.

Results No adverse events were reported during follow-up period. Comparing with baseline data, the level of $A$ poB decreased significantly at the end of the study $(0.589 \pm 0.136 \mathrm{~g} / \mathrm{l}$ vs $0.681 \pm 0.132 \mathrm{~g} / \mathrm{l}$, $\mathrm{p}=0.03)$. at 12 month IVUS follow-ups, minimal lumen CSA increased $\left((6.32+1.42) \mathrm{mm}^{2}\right.$ vs $\left.(4.63+0.51) \mathrm{mm}^{2}, \mathrm{p}<0.01\right)$, the plaque/media (P\&M) area decreased $\left(6.70 \pm 1.19 \mathrm{~mm}^{2}\right.$ vs $\left.8.17 \pm 1.55 \mathrm{~mm}^{2}\right), \mathrm{p}<0.05$; Plaque Burden decreased $(56.94 \pm 8.47 \%$ vs $61.4 \pm 10.34 \%, p<0.01)$. A total of 25 soft plaques $(50 \%)$ transformed into fibrous plaque. Comparing with baseline data, level of MMP-9 and hs-CRP decreased at the end of the study, $((1636+483) \mathrm{ng} / \mathrm{ml}$ vs $(2241+354) \mathrm{ng} / \mathrm{ml}, \mathrm{p}<0.001)$ and $((0.39+0.19 \mathrm{mg} / \mathrm{l}) \mathrm{vs}(3.48+1.50)$ $\mathrm{mg} / \mathrm{l}, \mathrm{p}<0.001)$, respectively. TIMP-1 increased $((788+110) \mathrm{ng} / \mathrm{ml}$ vs $(664+102) \mathrm{ng} / \mathrm{ml}, \mathrm{p}<0.001)$. In stepwise multivariate linear regression analysis, the only independent predictor of changes in P\&M area per year was decrement of MMP-9 and hs-CRP $(\gamma=0.85$, $\mathrm{p}<0.01$, and $\gamma=0.83, \mathrm{p}<0.01$, respectively). Regression equation is Annual Change of P\&M area $=-1.327+0.003$ Annual Change of MMP-9 +0.344 Annual Change of hs-CRP, R Square=0.830, Adjusted R Square $=0.819, \mathrm{~F}=78.152, \mathrm{p}=0.000$.

Conclusions Atrovastatin therapy stabilises borderline vulnerable plaque and reverses atherosclerosis progression in patients with ACS. Reversion of the atherosclerotic progression of vulnerable plaque is accompanied by the decrement of the level of plasma MMP-9 and hs-CRP. Changes in the level of MMP-9 and hs-CRP could predict the stabilisation of vulnerable plaque. 